\title{
OBSERVATION DE TUMEURS DU TRONC EN FORÊT DE FONTAINEBLEAU
}

\author{
M. WICKER
}

On peut observer sur le tronc de diverses essences de forêts ou de parcs, des tumeurs partois volumineuses. Une prospection commencée en forêt de Fontainebleau, dans le but initial de repérer du matériel d'étude, a montré que ces excroissances n'étaient pas rares. La recherche fut alors étendue dans cette forêt à des secteurs dont le peuplement, le relief et le sol étaient différents. Diverses mensurations concernant la morphologie et la position des tumeurs ont été collectées, afin de faire apparaître d'éventuelles différences dans le mode de croissance des tumeurs, suivant les essences sur lesquelles elles se développent.

\section{MATÉRIEL ET MÉTHODES}

A partir des routes forestières, les arbres sont d'abord repérés à vue, les tumeurs formant souvent des taches plus sombres sur l'ensemble des troncs. Cette opération est plus aisée en hiver et au printemps, en l'absence des feuillages et de la végétation des sous-bois. Chaque arbre est situé sur la carte par la mesure de ses distances à divers repères topographiques, essentiellement routes et chemins. L'essence de l'arbre est notée, ainsi que la position de la tumeur sur le tronc. Cinq mesures sont prises systématiquement pour chaque tumeur (figure 1):

- le diamètre du tronc à 1 mètre du sol (D) ;

- la distance de la tumeur au sol $(H)$;

- l'extension latérale (l) et verticale ( $h$ ) de la tumeur mesurées sur le tronc;

- le pourtour horizontal (L) de la tumeur à son extension maximum.

\section{Figure 1}

Différentes mesures prises sur les arbres

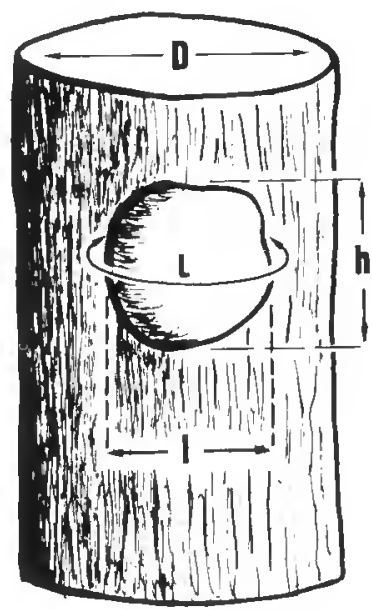




\section{RÉSULTATS}

\section{ESSENCES AFFECTÉES}

Au sein de chacune des principales essences de la forêt, on observe l'existence d'individus porteurs de tumeurs. Parmi les arbres recensés, les chênes figurent en plus grand nombre, mais ils constituent aussi l'essence la plus répandue dans la forêt (tableau 1). Par contre, les pins sylvestres porteurs de tumeurs sont relativement peu nombreux, alors qu'ils contribuent au peuplement d'ensemble dans une proportion presque équivalente à celle des chênes. Les bouleaux et les charmes sont relativement nombreux si l'on tient compte de leur faible représentation dans la population totale.

Tableau 1

Représentation des essences

parmi les arbres porteurs de tumeurs recensés

\begin{tabular}{|c|c|c|c|c|}
\hline Essences & $\begin{array}{l}\text { Arbres porteurs } \\
\text { d'une ou } \\
\text { plusieurs } \\
\text { tumeurs }\end{array}$ & $\begin{array}{c}\text { Pourcentage } \\
\text { représenté } \\
\text { dans l'échantillon } \\
\text { total }\end{array}$ & $\begin{array}{c}\text { Pourcentage } \\
\text { des essences } \\
\text { en forêt } \\
\text { de Fontainebleau }\end{array}$ & $\begin{array}{l}\text { Nombre total } \\
\text { de tumeurs } \\
\text { repérées }\end{array}$ \\
\hline Chênes & 112 & 62,6 & 44 & 157 \\
\hline Hêtres .......... & 30 & 16,8 & 10 & 35 \\
\hline Pins sylvestres & 13 & 7,3 & 41 & 29 \\
\hline Bouleaux $\ldots . .$. & 14 & 7,8 & & 15 \\
\hline Charmes & 8 & 4,5 & 5 & 8 \\
\hline Merisiers $\ldots . .$. & 2 & 1,1 & & 2 \\
\hline TOTAL & 179 & & & 246 \\
\hline
\end{tabular}

\section{MORPHOLOGIE DES TUMEURS}

\section{Description générale}

Les tumeurs sont généralement très saillantes, se raccordant souvent au tronc par un angle rentrant. Elles peuvent atteindre un développement très important sur de vieux arbres (Wicker, 1970). Certaines enveloppent complètement le tronc (planche II-figure 3). La surface est souvent régulière (planche 1 -figures $1,2,3$ ), mais peut aussi être bosselèe ou plus profondément divisée, la tumeur étant formée de plusieurs masses accolées (planche I-figure 4).

L'écorce présente un aspect variable selon les essences. Les tumeurs de chênes sont toujours recouvertes d'un rhytidome très épais et crevassé (Wicker, 1970). Les tumeurs de bouleaux portent souvent, elles aussi, une écorce épaisse (planche II-figure 3), les tumeurs de hêtres plus rarement. Dans ces deux dernières essences, l'écorce des tumeurs est, parfois sur les bouleaux (planche II-figure 4), et le plus souvent sur les hêtres, peu épaisse, fendillée ou lisse (planche 1-figure 3). Sur les tumeurs de charmes et de pins, l'écorce est peu différente de celle du tronc, légèrement plus épaisse et irrégulière (planche II, figures 1 et 2). 

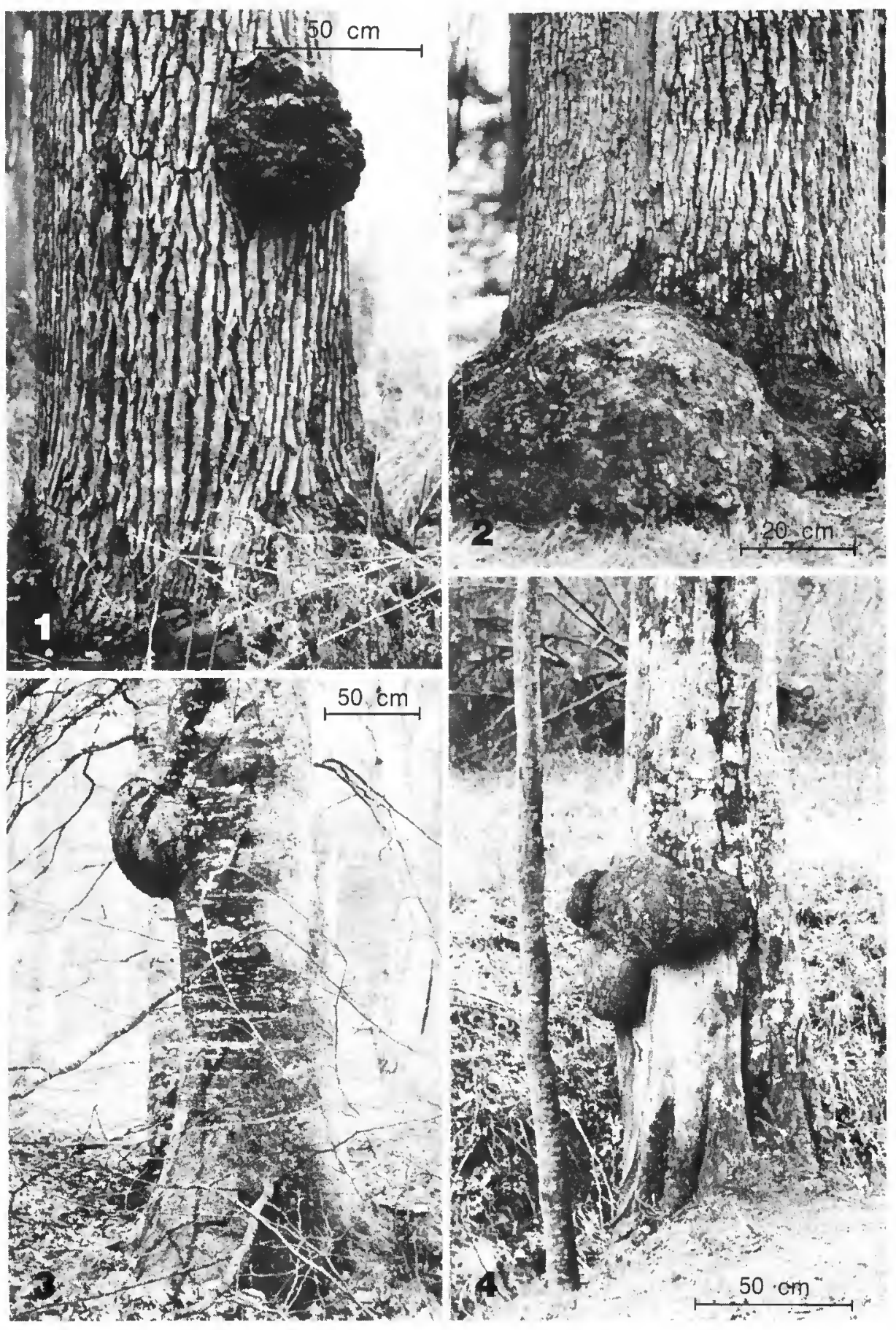

Planche 1

Exemples de tumeurs. 1 - sur le tronc d'un chêne rouvre; 2 - au ras du sol sur un chêne rouvre; 3 -à surface régulière sur le hêtre; 4 -mammelonnée sur le hêtre. 


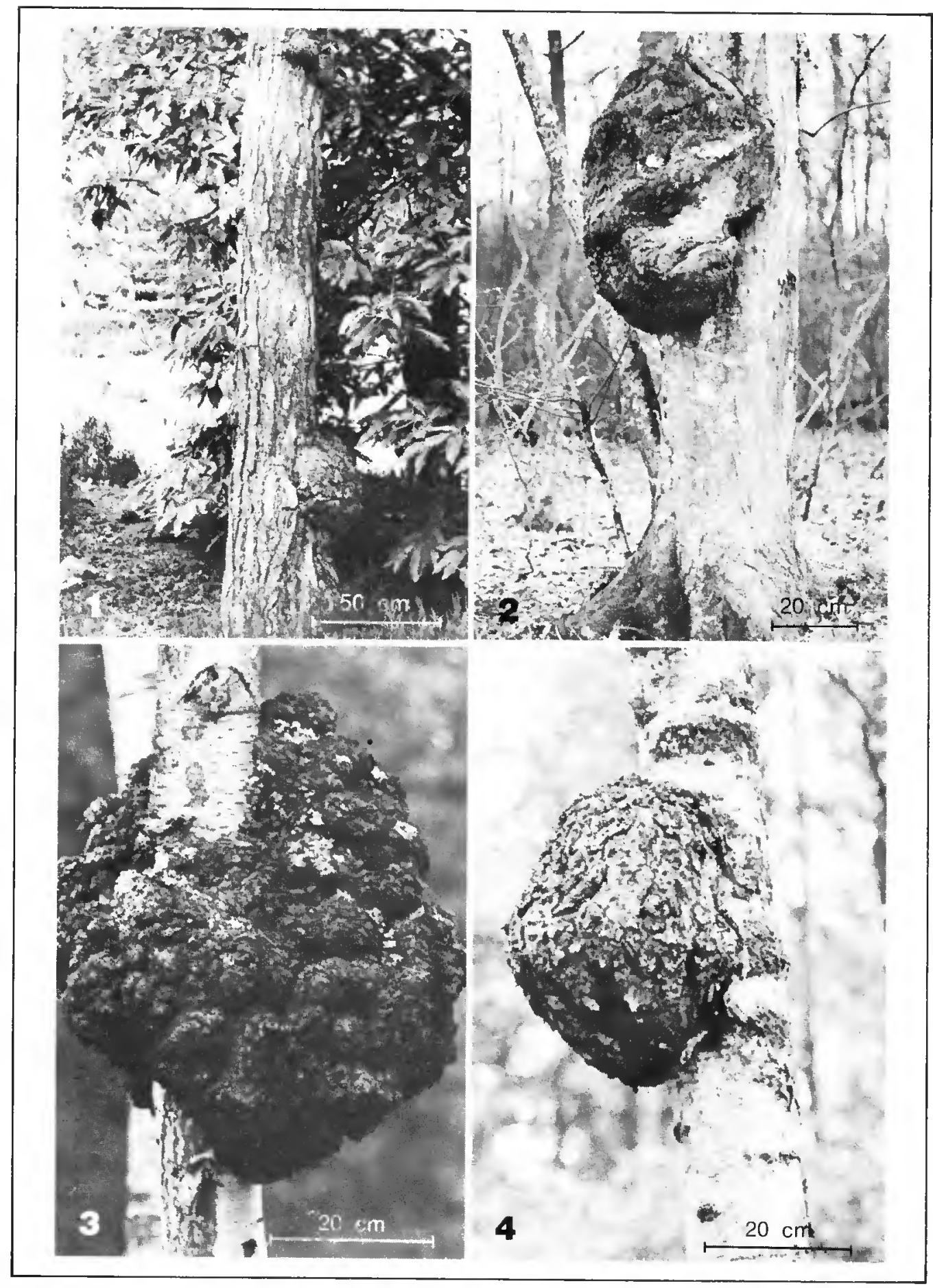

Planche 2

Exemples de tumeurs. 1 -plusieurs tumeurs sur un pin sylvestre; 2 -tumeur de charme; 3 et 4 -tumeurs de bouleaux. 


\section{Mensurations}

- Relation entre l'extension latérale (I) et verticale (h)

Le tableau 2 montre que dans les cinq essences concernées, à l'exception des charmes, un plus grand nombre de tumeurs manifeste un développement latéral plus important que le développement vertical.

Tableau 2

Classement des tumeurs par comparaison de leur extension latérale et verticale

\begin{tabular}{|l|c|c|c|}
\hline \multirow{2}{*}{ Essences } & \multicolumn{3}{|c|}{ Pourcentage } \\
\cline { 2 - 4 } & $1>\mathrm{h}$ & $1=\mathrm{h}$ & $1<\mathrm{h}$ \\
\cline { 2 - 4 } & & & \\
Chênes . & $78,0 \%$ & $10,0 \%$ & $12,0 \%$ \\
Hêtres .. & $59,0 \%$ & $20,5 \%$ & $20,5 \%$ \\
Pins syl- & & & \\
vestres .. & $87,0 \%$ & $4,3 \%$ & $8,6 \%$ \\
Bouleaux & $66,6 \%$ & $13,3 \%$ & $20,0 \%$ \\
Charmes . & $50,0 \%$ & $12,5 \%$ & $37,5 \%$ \\
\hline
\end{tabular}

Tableau 3

Relation entre le pourtour horizontal maximum des tumeurs et leur extension latérale sur le tronc

\begin{tabular}{|l|c|c|c|}
\hline \multirow{2}{*}{ Essences } & \multicolumn{3}{|c|}{ Pourcentage } \\
\cline { 2 - 4 } & $\begin{array}{c}\text { Tumeurs } \\
\text { aplaties }\end{array}$ & $\begin{array}{c}\text { Tumeurs } \\
\text { sphériques }\end{array}$ & $\begin{array}{c}\text { Tumeurs } \\
\text { saillantes }\end{array}$ \\
\hline Chênes . & $56 \%$ & $2 \%$ & $42 \%$ \\
Hêtres .. & $30 \%$ & $0 \%$ & $70 \%$ \\
Pins syl- & $52 \%$ & $4 \%$ & $44 \%$ \\
vestres .. & $5 \%$ & $0 \%$ & $93 \%$ \\
Bouleaux. & $7 \%$ & $0 \%$ & $100 \%$ \\
\hline Charmes . & $0 \%$ & $0 \%$ \\
\hline
\end{tabular}

\section{- Importance relative de la croissance radiale et latérale}

Dans le cas où la croissance est identique dans toutes les directions, la tumeur se présente comme une demi-sphère. $L$ correspond à la demi-circonférence de la sphère complète, I au diamètre, $\frac{L}{l}=1,57$ (figure 1). Lorsque $\frac{L}{l}<1,57$, l'écart entre les valeurs de $L$ et I est faible, les tumeurs sont aplaties. Lorsque $\frac{L}{l}>1,57$, l'écart entre les valeurs. de $L$ et I augmente, les tumeurs sont saillantes. Lorsqu'on classe les tumeurs en fonction de la valeur du rapport $\frac{L}{1}$ (tableau 3 ), on constate parmi les chênes et les pins une légère prédominance des tumeurs aplaties. Par contre, sur les hêtres, les bouleaux et les charmes on trouve une nette majorité de tumeurs proéminentes.

\section{- Relation entre le développement des tumeurs et le diamétre du tronc}

Les figures 2 et 3 montrent la distribution des points dont les coordonnées correspondent respectivement à $D$, le diamètre du tronc à 1 mètre du sol, et à $L$, le pourtour horizontal des tumeurs mesuré à leur maximum d'extension, parmi les quatre essences où des tumeurs ont été recensées en nombre suffisant pour faire une étude statistique: chênes, hêtres, pins sylvestres et bouleaux. Les quatre diagrammes de dispersion obtenus sont différents. L'existence d'une certaine dépendance entre les deux variables $D$ et $L$ peut être appréciée par le calcul du coefficient de corrélation $r$, qui fait intervenir la covariance et les écarts-types des deux séries de variables. Dans les quatre essences retenues, seul le coefficient de corrélation relatif aux tumeurs de chênes est significatif. Sa valeur $(r=0.62)$ indique une corrélation positive, c'est-à-dire qu'en général aux plus grandes valeurs de $\mathrm{D}$ correspondent les plus grandes valeurs de $\mathrm{L}$. Dans les trois autres 


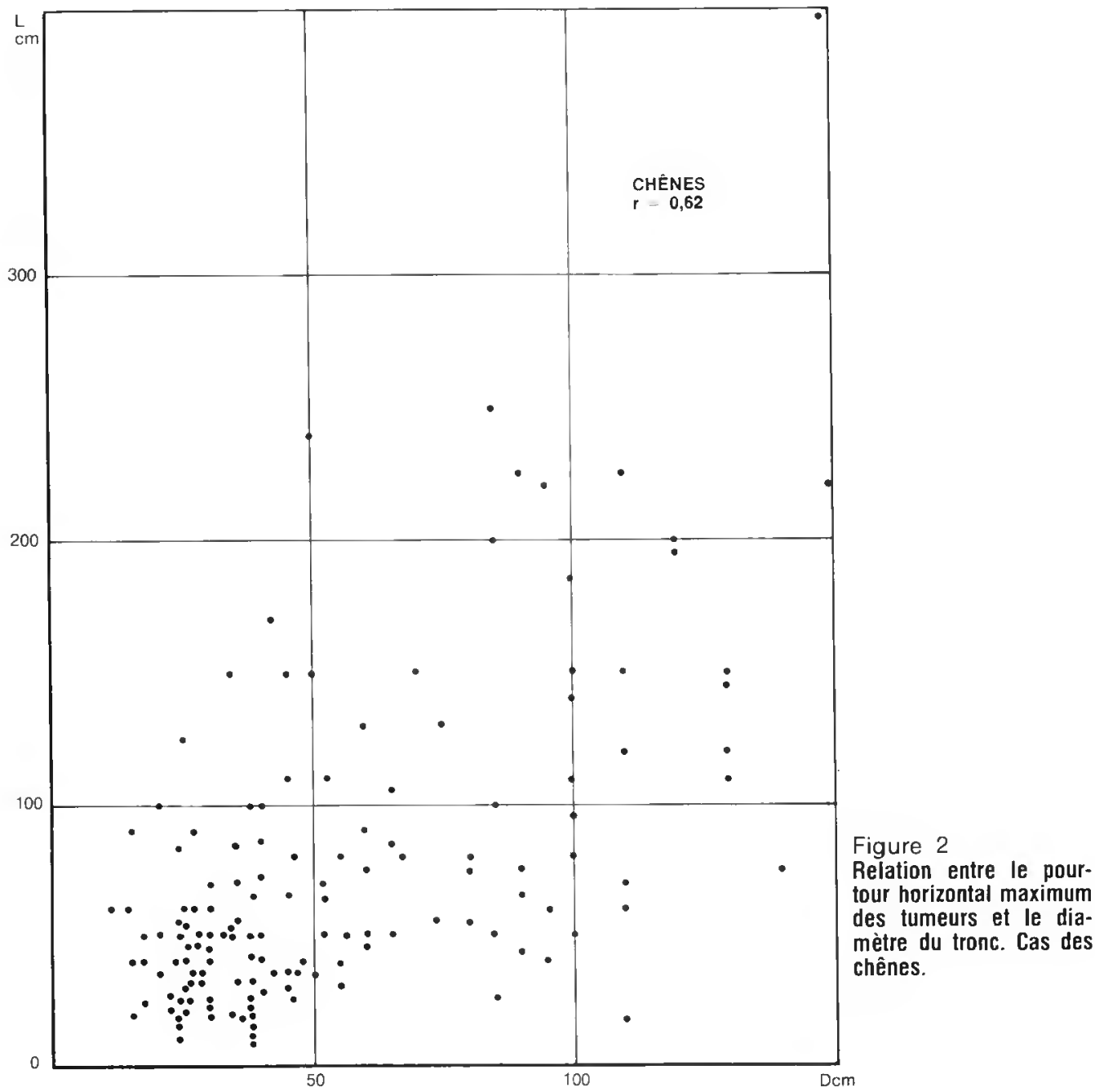

essences les valeurs de $r$ ne sont pas significatives, elles sont peut-être liées au hasard de l'échantillonnage, et à la faiblesse des effectifs (hêtres: 33 tumeurs; pins sylvestres : 23 tumeurs; bouleaux : 15 tumeurs).

\section{STRUCTURE DES TUMEURS}

Des sections transversales de tumeurs, observées à l'œil nu, montrent généralement des perturbations importantes de la structure du bois. On ne reconnaît pas toujours les cernes formés par les accroissements annuels. Lorsqu'ils sont visibles, leur tracé est irrégulier, ils sont souvent deux à trois fois plus larges que dans le tronc. Des nodules d'écorce interne sont fréquents. L'observation microscopique de coupes effectuées dans les trois plans transversal, tangentiel et radial, révèle les mêmes anomalies dans des tumeurs de chêne et de hêtre (Wicker, 1970). On retrouve tous les types cellulaires du bois, mais avec des dimensions et dans des proportions 

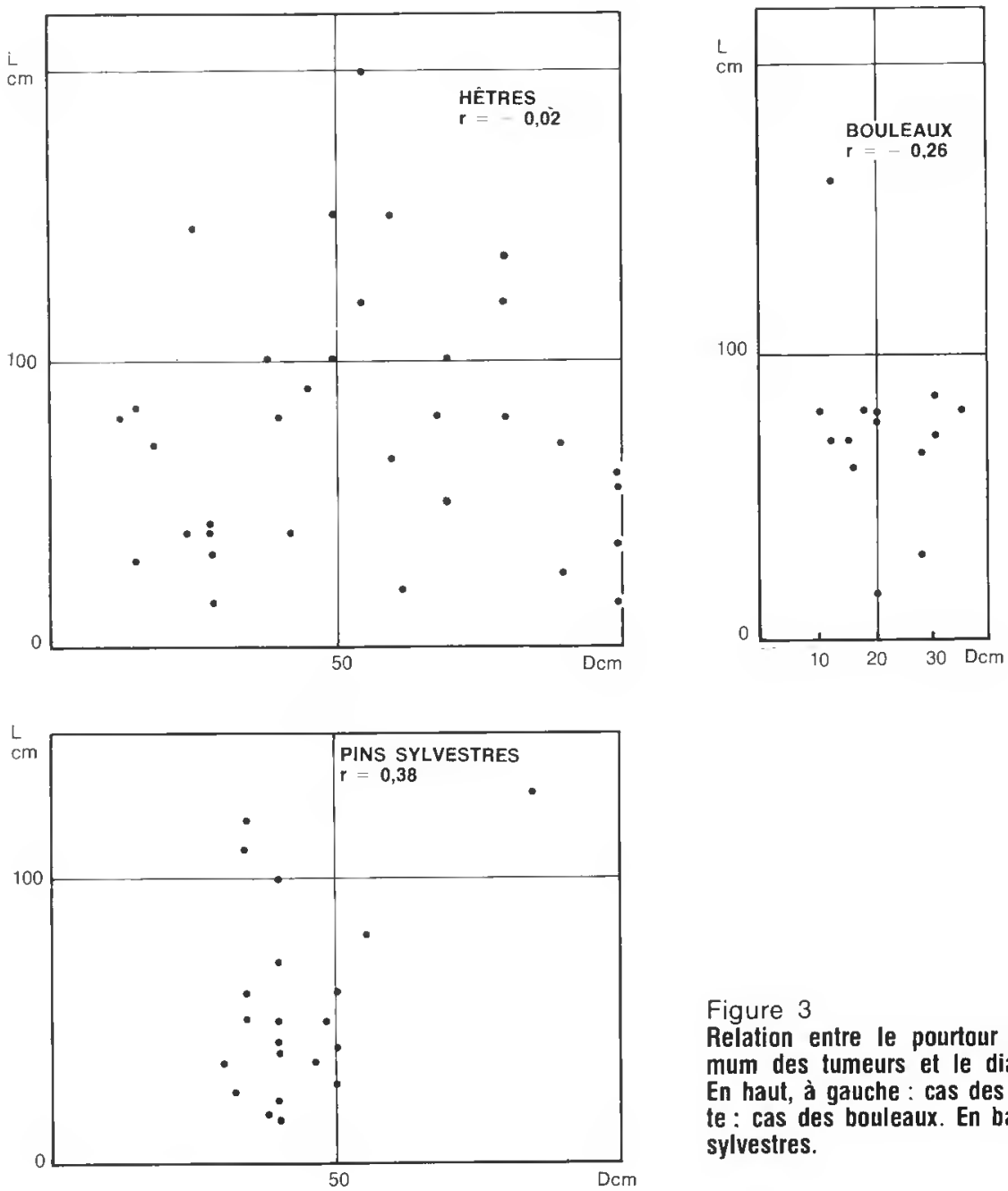

Figure 3

Relation entre le pourtour horizontal maximum des tumeurs et le diamètre du tronc. En haut, à gauche : cas des hêtres et à droite: cas des bouleaux. En bas: cas des pins sylvestres.

très modifiées. En coupe transversale, les vaisseaux, très peu nombreux, ont un diamètre beaucoup plus faible que dans le bois normal. En coupe tangentielle, on observe une invasion du bois par le parenchyme des rayons qui présentent une largeur uniforme. En coupe radiale, les cellules des rayons sont plus hautes et plus étroites que dans le bois normal. Les mêmes modifications s'observent dans les tumeurs de bouleaux. L'anatomie des tumeurs de pins n'a pas encore été examinée.

\section{LOCALISATION DES TUMEURS}

Situation et nombre des tumeurs sur les arbres

II s'agit presqu'uniquement d'excroissances portées par les troncs. Les racines n'ont pas pu être observées. La figure 4 montre que le plus grand nombre de tumeurs (82\%) se trouve à moins de 4 mètres du sol. On en observe cependant quelques-unes jusqu'à 12 et 15 mètres 


\section{WICKER}

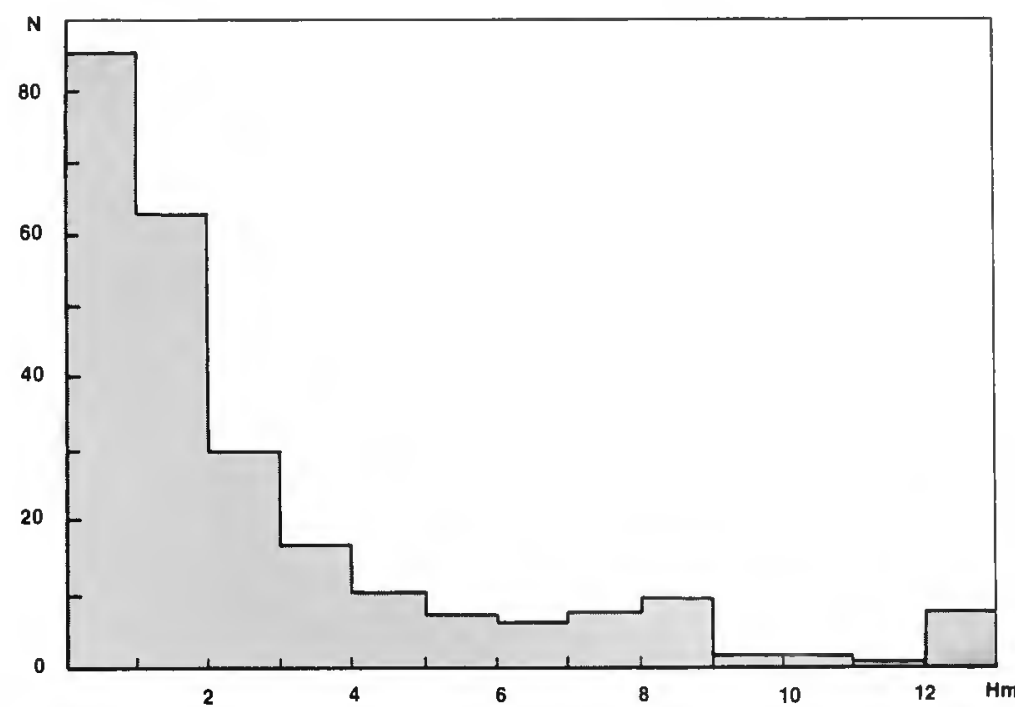

Figure 4

Situation des tumeurs au-dessus du sol

au-dessus du sol, sur de vieux arbres. Parmi les 232 tumeurs ayant fait l'objet de mesures, 11 seulement, soit $4,7 \%$ se situent au ras du sol. Il n'a étè observé que deux tumeurs sur des branches, l'une sur un chêne, l'autre sur un hêtre. Que les tumeurs soient solitaires ou plusieurs sur un même sujet, leur implantation sur l'arbre semble se faire au hasard, sans qu'il soit possible de mettre en évidence une orientation privilégieee.

Certains arbres portent plusieurs tumeurs, mais celles-ci ne sont jamais en très grand nombre, contrairement à ce que White a observe sur Picea glauca (White et al., 1954). Les tableaux 1 et 4 montrent que parmi 179 arbres recensés, 36 soit $20 \%$ sont porteurs de plusieurs tumeurs, le nombre maximum de tumeurs par arbre étant de 7. Ce sont les chênes (28) et les pins sylvestres (4) qui manifestent le plus souvent cette particularité.

Tableau 4 Répartition des arbres suivant le nombre de tumeurs portées, dans les différentes essences

\begin{tabular}{|c|c|c|c|c|c|c|c|}
\hline \multirow[b]{2}{*}{ Essences } & \multicolumn{7}{|c|}{ Arbres porteurs de } \\
\hline & $\begin{array}{c}1 \\
\text { tumeur }\end{array}$ & $\begin{array}{c}2 \\
\text { tumeurs }\end{array}$ & $\begin{array}{c}3 \\
\text { tumeurs }\end{array}$ & $\begin{array}{c}4 \\
\text { tumeurs }\end{array}$ & $\begin{array}{c}5 \\
\text { tumeurs }\end{array}$ & $\begin{array}{c}6 \\
\text { tumeurs }\end{array}$ & $\begin{array}{c}7 \\
\text { tumeurs }\end{array}$ \\
\hline Chênes & 84 & 20 & 4 & 1 & 1 & 2 & 0 \\
\hline Hêtres & 27 & 2 & 0 & 1 & 0 & 0 & 0 \\
\hline Pins sylvestres & 9 & 1 & 0 & 1 & 0 & 0 & 2 \\
\hline Bouleaux & 13 & 1 & 0 & 0 & 0 & 0 & 0 \\
\hline Charmes ... & 8 & 0 & 0 & 0 & 0 & 0 & 0 \\
\hline Merisiers ..... & 2 & 0 & 0 & 0 & 0 & 0 & 0 \\
\hline
\end{tabular}

\section{Répartition des arbres affectés dans la forêt}

- Influence de la nature géologique du sol

Deux types de relief s'opposent à Fontainebleau et correspondent à deux sortes de sols: les plaines basses ou hautes, formées par l'affleurement du calcaire de Brie (Sannoisien) ou du 
calcaire de Beauce (Aquitanien); entre ces deux dépôts de calcaires lacustres, l'épaisse couche des sables et grès de Fontainebleau (Stampien). On trouve cette dernière sur les pentes menant des basses plaines aux hautes plaines, ou en dunes formant les différents "rochers", là où le calcaire de Beauce a été détruit par l'érosion. Des blocs de grès couvrent les pentes et les sommets de ces dunes.

Sur le calcaire et le sable, en plaines basses, les chênes et les hêtres prédominent; dans les "rochers " ou sur les pentes menant aux hautes plaines, les pins et les bouleaux se substituent en partie ou totalement aux essences précédentes.

Les arbres porteurs de tumeurs se rencontrent aussi bien sur sol calcaire que sur sol sableux, cependant sur le sable des "rochers" ils sont rares. Deux différences entre les plaines et les "rochers " sont peut-être à considérer : une possible hydratation plus faible du sol des "rochers", et un peuplement constitué généralement d'arbres plus jeunes que dans la plupart des zones de plaines.

\section{- Répartition par zones}

Bien que certains arbres porteurs de tumeurs soient isolés, la plupart sont groupés, et leur répartition sur la carte dessine nettement des taches. Dans tous les secteurs traités actuellement en réserves biologiques, ou ayant constitué les réserves artistiques supprimées en 1967, les tumeurs sont fréquentes. Ainsi en est-il du Gros Fouteau (figure 5), réserve biologique; du Bas

Figure 5

Répartition géographique en tache des arbres porteurs de tumeurs dans un secteur traité en réserve biologique

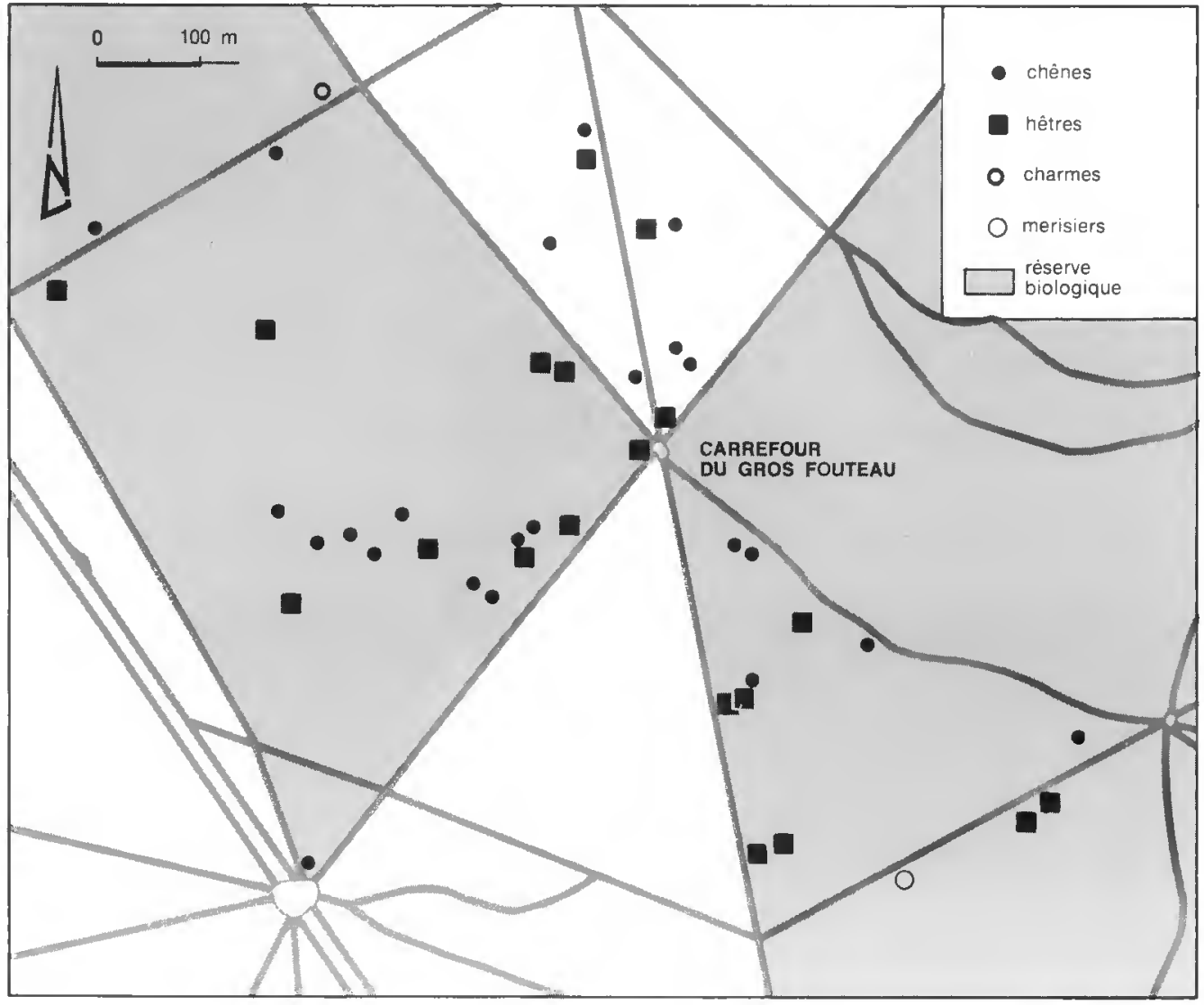




\section{WICKER}

Bréau et des Ventes à la Reine, anciennes réserves artistiques. On y trouve de très vieux arbres, et parmi eux, des porteurs de tumeurs. Ceux-ci niont pas éte éliminés par les coupes, car l'exploitation de ces secteurs est suspendue depuis plus d'un siècle. Ailleurs il est vraisemblable que le port d'une tumeur désigne l'arbre pour la coupe. II s'agit en effet d'un défaut dont on peut redouter qu'il soit héréditaire. Mais ce n'est pas dans les seules réserves que l'on constate une concentration de porteurs de tumeurs par taches. La figure 6 montre une zone plate à l'est de la route Lorentz, établie sur les sables stampiens, occupée par une futaie de chênes, puis à l'ouest les pentes qui ménent au calcaire de Beauce, oủ les pins sylvestres se mêlent aux chênes. On remarque deux zones de part et d'autre de la route Lorentz, oủ les porteurs de tumeurs sont plus fréquents.

Figure 6

Exemples de répartition en tache des arbres porteurs de tumeurs dans une zone d'exploitation traditionnelle

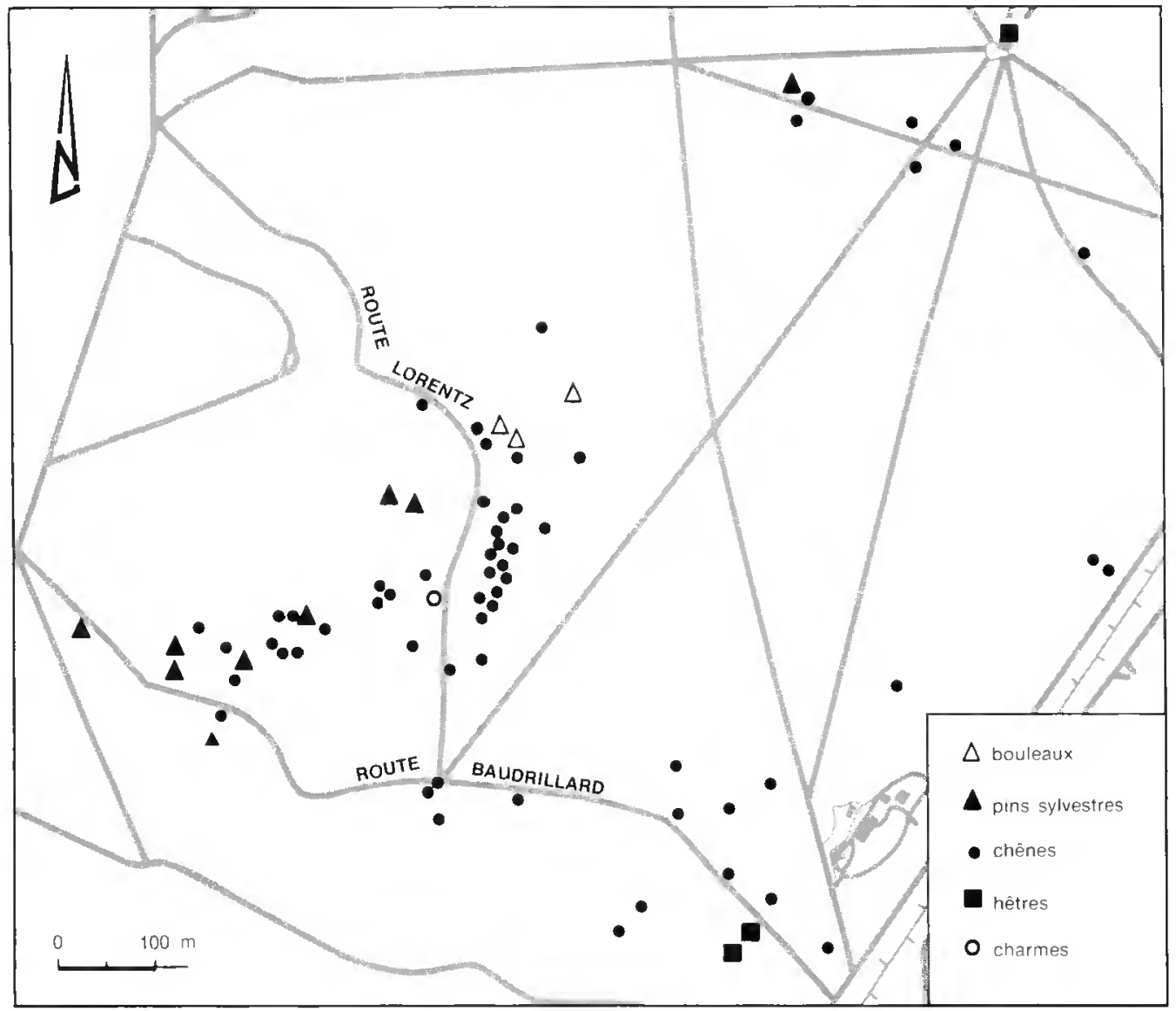

\section{DISCUSSION ET CONCLUSION}

Ces tumeurs du tronc ne sont pas une exclusivité de la forêt de Fontainebleau, il y en a dans d'autres forêts du Bassin parisien, et certainement dans toute la France. Dufrenoy (1925) a décrit des tumeurs sur divers résineux de France et du nord de l'Italie. Elles ne sont pas 
particulières aux essences forestières de nos régions, certains arbres originaires d'autres pays et introduits en France peuvent en porter. Nous en avons ainsi observé en région parisienne sur Paulownia, platane, cerisier du Japon et cyprès chauve de Louisiane. Des tumeurs semblables ant été signalèes en Amèrique du Nord sur des feuillus (Brown, 1936, 1938, 1941), sur des résineux (White et Millington, 1954; White, 1958; Peterson, 1961; Ouellette, 1977; Rickey et al., 1974), en Allemagne sur Sequoia (Martin, 1957), sur des feuillus et des résineux (Sprengel, 1936). On en trouve ègalement en Australie sur les Eucalyptus. Étant donnée cette grande variété d'hôtes et de situations géographiques, il est peu probable que toutes ces proliférations aient la même origine. Dans certains cas, le rôle d'un organisme pathogène a pu être mis en évidence, il s'agit soit d'un champignon du genre Phomopsis isolé des tumeurs étudiées par Brown, soit de diverses bactèries, isolèes de tumeurs de résineux (Dufrenoy, 1925 ; Eskandari et Ridé, 1967 sur le Pin d'Alep; Ride sur le Pin Cembro, communication personnelle). Dans le cas des tumeurs ligneuses du sapin, désignées en France par les noms de "dorge " ou de "chaudron ", suivant les régions, l'agent pathogène et son cycle biologique sont parfaitement connus; il s'agit d'une Rouille, Melampsorella caryophyllacearum (Guinier, 1922). La morphologie de ces tumeurs est plus variable et assez différente de celle des exemples précédents. D'autre part, ces déformations sont liées aux balais de sorcière. On peut en réduire l'importance de façon très satisfaisante en supprimant les balais de sorcière, et en èliminant les arbres atteints de chaudron. L'apparition de beaucoup d'autres tumeurs reste cependant sans cause décelable (Sprengel, 1936; Bull, 1951 , White, 1958 ; Peterson, 1961; Ouellette, 1967). Les observations réunies par cette étude suggèrent quelques remarques.

La présence de tumeurs sur les principales essences du peuplement de Fontainebleau, y compris des essences faiblement représentées, comme le merisier ou le charme, montre que le phenomène n'est pas particulier à l'une d'elles. L'échantillon d'arbres du tableau 1 n'est pas quelconque puisqu'il rassemble des arbres porteurs d'une ou plusieurs tumeurs. II n'est certainement pas représentatif du peuplement total, et ne peut lui être strictement comparé. Mais dans l'hypothèse où les tumeurs recensėes auraient la même origine, il serait intéressant de chercher si les diverses essences qui les portent présentent une sensibilité particulière à l'agent tumorigène. Les données du tableau 1 sont insuffisantes à cet égard, et nous pensons revoir ce problème, dans des parcelles où des arbres porteurs de tumeurs ont été repérés, et pour lesquelles des données d'inventaires par essence sont connues.

L'ètude morphologique des tumeurs donne des indications sur le fonctionnement du cambium. D'une part, dans chacune des cinq essences concernées, le plus grand nombre de tumeurs presente une extension latèrale supérieure à l'extension verticale. L'ètude microscopique de bois de tumeurs et de bois normal de chênes et de hêtres montre en effet, en coupes tangentielles, un envahissement du bois par le parenchyme des rayons (Wicker, 1970). D'autre part, le rapport entre le pourtour horizontal maximum des tumeurs et leur extension latérale sur le tronc est diffèrent selon les essences. Cette variabilitè morphologique reflète une dysharmonie dans les rythmes des divisions des initiales cambiales dans chacun des plans transversal, radial ou tangentiel.

L'existence ou l'absence de corrélation entre le pourtour horizontal maximum des tumeurs et le diamètre du tronc mesuré à 1 mètre du sol pourrait donner une indication sur le stade de développement de l'arbre où l'initiation du processus tumoral est la plus fréquente. Cependant on n'a trouvè une corrélation positive significative entre $L$ et $D$ que pour les chênes, parmi les quatre essences étudiées, ce qui revient à dire que l'on trouve généralement dans ce groupe, les plus grosses tumeurs sur les plus gros arbres. On trouve néanmoins de petites tumeurs sur de gros chẻnes, ce qui montre que cette approche du problème permet uniquement de dégager une tendance gènérale. Il est certain que seul l'examen de la section transversale du tronc au niveau de la tumeur, dans chaque cas, peut nous renseigner sur le début de son développement. 
Le faít que les tumeurs se trouvent plus fréquemment à moins de 4 mètres du sol est à rapprocher de l'observation analogue faite par Jacquiot et Viney (1954) sur la " graisse du chêne". Ou bien la région de l'atmosphère concernée réunit des conditions plus favorables au développement des tumeurs, parmi lesquelles on peut évoquer le degré hygrométrique, la fréquentation peut-être plus grande par des insectes vecteurs de micro-organismes ou de virus, les occasions plus fréquentes de blessures par l'homme ou les animaux; ou bien les tumeurs surviennent et se développent le plus souvent dès le début de la vie des arbres.

La distribution fréquente des arbres affectés par zones évoque un phénomène parasitaire ou génétique. Le crown gall est parmi les tumeurs végétales d'origine bactérienne, l'une des plus connues. Induit par l'Agrobacterium tumefaciens, qui est une bactérie du sol, il se développe généralement au niveau du collet. Or la proportion de tumeurs que nous avons observées au ras du sol est très faible $(4,7 \%)$. Par ailleurs, le crown gall semble peu répandu sur les essences forestières (Ridé et Morand, 1976). Des recherches d'organismes pathogènes, connus comme agents d'autres types de proliférations tumorales, n'ont pas abouti. Aucun des champignons ou des bactéries que nous avons isolés de tumeurs, n'ont permis d'obtenir de semblables excroissances par inoculation à des plantes saines $\left({ }^{\circ}\right)$. La présence de champignons ou de bactéries dans les coupes microscopiques de tumeurs réalisées jusqu'à présent n'est pas évidente. Actuellement, aucun de nos résultats ne permet de conclure que ces tumeurs sont liées à la présence d'un agent parasitaire. L'hypothèse d'un phénomène génétique reste à étudier.

Ces résultats font apparaître la nécessité de poursuivre la comparaison du développement et de la structure des tumeurs du tronc, afin de mieux cerner l'existence de variations liées à l'essence de l'arbre, ou aux facteurs de l'environnement. L'extension de cette étude est prévue dans deux autres forêts d'lle-de-France.

(") A ce sujet, il nous serait très utile de pouvoir recolter de petites tumeurs (diametre maximum: 5 a $8 \mathrm{~cm}$ ), que des lecteurs pourraient nous signaler, ou des tumeurs plus grosses portees par des arbres devant être abattus. 


\section{RÉFÉRENCES BIBLIOGRAPHIQUES}

BROWN (N.A.). - Privet and Jasmine galls produced by a species of Phomopsis. Phytopathology, 26, 1936 , pp. 795-799.

BROWN (N.A.). - The tumor disease of Oak and Hickory tress. Phytopathology, 28, 1938, pp. 401-411.

BROWN (N.A.). - Tumors on elm and maple trees. Phytopathology, 31, 1941, pp. 541-548.

BULL (R.A.). - A new gall disease of Sequoia sempervirens. Gard. chron., 130, 1951, pp. 110-111.

DUFRENOY (J.). - Les tumeurs des résineux. Annales de l'Institut national d'Agronomie, 19, 1925, pp. 33-201.

ESKANDARI (F.). - Acquisitions nouvelles concernant les tumeurs du Pin d'Alep. Thèse de Docteur-Ingènieur, Paris, 1967.

ESKANDARI (F.), RIDE (M.). - Etiologie des tumeurs du Pin d'Alep (Pinus halepensis Mill), comptes rendus de l'Académie des Sciences, 264, 1967, pp. 2638-2641.

GUINIER (P.). - La "dorge " ou "chaudron" du Sapin. Bulletin de la Socièté forestière de Franche-Comté et Belfort, 14, 1922, pp. 333-347.

JACQUIOT (C.). VINEY (R.). - La graisse du chêne. Revue de Pathologie végétale et d'entomologie appliquée, XXXIII, $n^{\circ} 2,1954$, pp. 65-79.

LAMOTTE (M.). - Initiation aux mèthodes statistiques en biologie. Paris, Masson Ed., 1957, pp. 112•135.

MARTIN (E.I.), - Neoplastiches Wachstum bei Sequoiadendron giganteum Buchholz. Phytopathologische Zeitschrift, 30, 1957, pp. 342-343.

OUELLETTE (G.-B.). - Sur l'origine et le développement de tumeurs chez l'épinette. Phytoprotection, $48, n^{\circ} 3$. 1967, pp. 101-106.

PETERSON (R.S.). - Conifer tumors in the central Rocky Mountains. Plant disease reporter, 45, $n^{\circ} 6,1961$, pp. $472-474$.

RICKEY (R.G.), HAMILTON (J.K.), HERGERT (H.L.). - Cheminal and physical properties of tumor-affected Sitka Spruce. Wood and fiber, 6, $n^{\circ} 3,1974$, pp. 200-210.

RIDE (M.), MORAND (J.-C.). - Bactérioses, viroses et mycoplasmoses forestiéres in LANIER (L.), JOLY (P.), BONDOUX (P.), BELLEMÉRE (A.). - Mycologie et pathologie forestieres. Tome II, Paris, Masson Ed. 1976, pp. 200-202.

SPRENGEL (F.). - Über die Kropfkrankheit an Eiche, Kiefer, und Fichte. Phytopathologische Zeitschrift, Bd 9 , Hft 6, 1936, pp. 583-635.

WHITE (P.R.). - A tree tumor of unknown origin. Procedings of the National Academy of Sciences of the U.S.A., $44, n^{\circ} 4,1958, p p .339-344$.

WHITE (P.R.), MILLINGTON (W.F.). - The distribution and possible importance of a woody tumor on trees of the White Spruce. Picea glauca. Canc. Res. 14, $n^{\circ} 2,1954$, pp. 128-134

WHITE (P.R.), MILLINGTON (W.F.). - The structure and development of a woody tumor affecting Picea glauca. American Journal ot Botany, 41. $n^{\circ}$ 4. 1954, pp. 353-361.

WICKER (M.). - Structure et densitè de bois de tumeurs de chêne et de hêtre. Revue génèrale de botanique, 77. 1970. pp. 499-517. 